ANNALES

POLONICI MATHEMATICI

$85.3(2005)$

\title{
Approximation by weighted polynomials in $\mathbb{R}^{k}$
}

\author{
by Maritza M. Branker (Toronto)
}

\begin{abstract}
We apply pluripotential theory to establish results in $\mathbb{R}^{k}$ concerning uniform approximation by functions of the form $w^{n} P_{n}$ where $w$ denotes a continuous nonnegative function and $P_{n}$ is a polynomial of degree at most $n$. Then we use our work to show that on the intersection of compact sections $\Sigma \subset \mathbb{R}^{k}$ a continuous function on $\Sigma$ is uniformly approximable by $\theta$-incomplete polynomials (for a fixed $\theta, 0<\theta<1$ ) iff $f$ vanishes on $\theta^{2} \Sigma$. The class of sets $\Sigma$ expressible as the intersection of compact sections includes the intersection of a symmetric convex compact set with a single orthant.
\end{abstract}

1. Introduction. Let $w$ be a nonnegative, continuous function on a closed set $\Sigma \subset \mathbb{R}^{k} \subset \mathbb{C}^{k}, k>1$, with the set $\{z \in \Sigma: w(z)>0\}$ not pluripolar. If $\Sigma$ is unbounded we also assume that $|z| w(z) \rightarrow 0$ as $|z| \rightarrow \infty$ for $z \in \Sigma$. Then $w$ is an admissible weight function. Given a continuous function $f$ on $\Sigma$, we consider when $f$ will be the uniform limit of functions of the form $w^{n} P_{n}$ where $P_{n}$ is a polynomial of degree at most $n$. We refer to a function of the form $w^{n} P_{n}$ as a weighted polynomial. This problem has been studied extensively in $\mathbb{R}$; for an introduction see [9]. Applying the Stone-Weierstrass theorem gives

THEOREM. Given an admissible continuous weight function $w$ on a closed set $\Sigma \subset \mathbb{R}^{k}$ there exists a closed set $Z=Z(w) \subset \Sigma$ such that a continuous function fon $\Sigma$ is the uniform limit of a sequence $\left\{w^{n} P_{n}\right\}_{n=1}^{\infty}$ of weighted polynomials if and only if $f$ vanishes on $Z$.

Therefore we need to find $Z(w)$ in order to solve the approximation problem. We consider $\Sigma$ as a subset of $\mathbb{C}^{k}$ and define the weighted pluricomplex Green function $V_{\Sigma, Q}$ where $Q:=-\log w$. When $w \equiv 1$ and $\Sigma$ is compact this will be the usual pluricomplex Green function. A natural step

2000 Mathematics Subject Classification: Primary 32U35; Secondary 41A10.

Key words and phrases: multivariable incomplete polynomials, weighted pluricomplex Green function.

Thesis work under the supervision of Professor T. Bloom at the University of Toronto. 
when calculating $Z(w)$ is to find the support $S_{w}$ of the equilibrium measure $\left(d d^{c} V_{\Sigma, Q}^{*}\right)^{k}$ since we have

TheOREM. Let $\Sigma \subset \mathbb{R}^{k}$ be a closed set which is not pluripolar in a neighbourhood of any of its points. Given a continuous admissible weight $w$ on $\Sigma$, a sequence $\left\{w^{n} P_{n}\right\}_{n=1}^{\infty}$ of weighted polynomials which converges uniformly on $S_{w}$ will tend to zero for every point in $\Sigma \backslash S_{w}$.

Our attention is focused on sets for which the boundary may be described using linear functions or positive quadratic forms. Then we have an associated radial weight function; for example considering the unit ball in $\mathbb{R}^{k}$ gives

Theorem. Let $\Sigma=\left\{x \in \mathbb{R}^{k}:|x| \leq 1\right\}$ with the weight $w=|x|^{\alpha}$ for $\alpha>0$. A continuous function $f$ on $\Sigma$ is the uniform limit of weighted polynomials $w^{n} P_{n}, n=1,2, \ldots$, if and only if $f$ vanishes on the set $\left\{x \in \mathbb{R}^{k}\right.$ : $|x| \leq \alpha /(1+\alpha)\}$.

Here $|\cdot|$ denotes the Euclidean norm in $\mathbb{C}^{k}$.

Our work with linear weights leads to an application to the theory of multivariable incomplete polynomials. In [7] G. G. Lorentz defined an incomplete polynomial to be of the form

$$
I_{n}(t)=\sum_{j=s}^{n} a_{j} t^{j}
$$

with a zero of order $s>0$ on the interval $[0,1]$. If $a_{s} \neq 0, a_{n} \neq 0$ the ratio $\tau=s / n$ is called the type of $I_{n}(t)$. Lorentz established many basic properties of incomplete polynomials including the fact that if for a sequence $\left\{I_{n}(t)\right\}$ of incomplete polynomials one has $\left|I_{n}(t)\right| \leq 1$ on $[0,1]$ and $s \geq \theta n$ for some $\theta \in(0,1)$ then $\lim _{n \rightarrow \infty} I_{n}(t)=0$ on $\left[0, \theta^{2}\right)$. In approximation theory, incomplete polynomials have been a subject of intense study. It was shown independently by Saff and Varga in [10] and v. Golitschek in [2] that using incomplete polynomials of type $\geq \theta$, for a fixed $\theta$, it is possible to uniformly approximate any function $f \in C[0,1]$ with the property $f \equiv 0$ on $\left[0, \theta^{2}\right]$. In [5] Kroó succeeded in establishing results concerning incomplete polynomials on a restricted class of convex domains in $\mathbb{R}^{2}$. However his methods are not easily generalized to higher dimensions. We define a compact section in Section 3 and then establish the following

TheOREm. Let $\Sigma$ be the intersection of compact sections in $\mathbb{R}^{k}$. Then it is possible to uniformly approximate a continuous function $f$ on $\Sigma$ by $\theta$-incomplete polynomials iff $f$ vanishes on $\theta^{2} \Sigma$.

For example, suppose we have a symmetric convex compact set $K$ in $\mathbb{R}^{k}$. Let $\Sigma$ denote the portion of $K$ which lies in the first orthant, that is, 
$\Sigma:=K \cap\left(\mathbb{R}^{+}\right)^{k}$. Then $\Sigma$ is the intersection of compact sections and the above theorem applies.

This paper was significantly influenced by the work done on the weighted approximation problem in the one-dimensional setting by Kuijlaars, Mhaskar, Saff and Varga, and Totik in [6], [8], [10], [12]. For a systematic development of weighted potential theory and its applications see the monograph [9].

The technique of proof used for many of our results utilizes weighted pluripotential theory, first introduced by Siciak [11]. The basic theory, summarized in Section 2, was done by Bloom in Appendix B of [9] and further developed in the recent paper of Bloom and Levenberg [1]. The one variable theory has already proven useful in tackling a variety of problems. It is hoped that as weighted pluripotential theory is further developed, the same will prove true in higher dimensions. The basic theory as well as some new weighted generalizations of pluripotential theoretic results can be found in Section 2. Finally, some of the results in Section 4 are generalizations of Kroó's work in $\mathbb{R}^{2}$ which may be found in [5].

2. Weighted pluripotential theory. We start by recalling the basic definitions and theorems of weighted pluripotential theory. This material, along with complete proofs, may be found in Appendix B of [9].

Let $\Sigma \subset \mathbb{C}^{k}$ be any set and $w$ a real-valued function on $\Sigma$ such that $w \geq 0$. The function $w$ is called a weight function or more simply a weight. For the duration of this paper we restrict our attention to admissible weights. Before giving the definition of an admissible weight we recall a few standard terms. Let $G$ denote an open set in $\mathbb{C}^{k}$.

Definition 2.1. A function $u: G \rightarrow[-\infty, \infty)$ is upper semicontinuous on $G$ if for every $z^{0} \in G, \lim \sup _{z \rightarrow z^{0}} u(z) \leq u\left(z^{0}\right)$.

Definition 2.2. A function $u: G \rightarrow[-\infty, \infty)$ is plurisubharmonic (psh) if it is upper semicontinuous on $G, u \neq-\infty$ on any connected component of $G$ and for every $a \in G, b \in \mathbb{C}^{k}$ the function of the single complex variable $\lambda \mapsto u(a+\lambda b)$ is subharmonic or identically $-\infty$ on every component of the set $\{\lambda \in \mathbb{C}: a+\lambda b \in G\}$.

We say a set $E \subset \mathbb{C}^{k}$ is pluripolar if for each point $a \in E$, there is a neighbourhood $B$ of $a$ and a plurisubharmonic function $u$ on $B$ such that $E \cap B \subset\{z \in B: u(z)=-\infty\}$. We also say that a property holds quasi-everywhere (q.e.) on a set $E$ if it holds on $E \backslash F$, where $F$ is pluripolar.

Definition 2.3. A weight function $w$ is admissible if it satisfies the following: 
(i) $w$ is upper semicontinuous;

(ii) the set $\{z \in \Sigma: w(z)>0\}$ is not pluripolar;

(iii) if $\Sigma$ is unbounded then $|z| w(z) \rightarrow 0$ as $|z| \rightarrow \infty$ for $z \in \Sigma$.

We define $Q=Q_{w}:=-\log w$.

Then the weighted pluricomplex Green function of $\Sigma$ with respect to $Q$ is defined for $z \in \mathbb{C}^{k}$ by

$$
V_{\Sigma, Q}(z):=\sup \{u(z): u \in \mathcal{L}, u \leq Q \text { on } \Sigma\}
$$

where

$$
\mathcal{L}:=\left\{u \in \operatorname{PSH}\left(\mathbb{C}^{k}\right): u \leq \log ^{+}|z|+C\right\}
$$

and $C$ is a constant which may depend on $u$. We denote the upper semicontinuous regularization of $V_{\Sigma, Q}$ by $V_{\Sigma, Q}^{*}$. Observe that for $w \equiv 1$ on compact $\Sigma$ this definition coincides with the usual pluricomplex Green function. For $\varrho>0$ we define $\Sigma_{\varrho}:=\{z \in \Sigma:|z| \leq \varrho\}$.

Lemma 2.4. For $\Sigma$ closed and $\varrho$ sufficiently large, $V_{\Sigma, Q}=V_{\Sigma_{\varrho}, Q}$.

Therefore the Borel measure $\left(d d^{c} V_{\Sigma, Q}^{*}\right)^{k}$ has compact support since it is equal to $\left(d d^{c} V_{\Sigma_{\varrho}, Q}^{*}\right)^{k}$ whose support is contained in $\Sigma_{\varrho}$. We will use the notation

$$
\mu_{w}:=\left(d d^{c} V_{\Sigma, Q}^{*}\right)^{k}, \quad S_{w}:=\operatorname{supp}\left(\mu_{w}\right), \quad S_{w}^{*}=\left\{z \in \Sigma: V_{\Sigma, Q}^{*}(z) \geq Q(z)\right\} .
$$

LEMma 2.5. Let $\Sigma \subset \mathbb{C}^{k}$ be a closed set and $w$ an admissible weight function. Then:

(i) $S_{w}$ is not pluripolar;

(ii) $S_{w} \subset S_{w}^{*}$;

(iii) $V_{\Sigma, Q}^{*} \leq Q$ q.e. on $\Sigma$;

(iv) $V_{\Sigma, Q}=Q$ for q.e. $z \in S_{w}\left(\right.$ or $\left.S_{w}^{*}\right)$.

We also have the following estimates involving weighted polynomials which will be used to prove Theorem 3.1. A weighted polynomial is of the form $w^{n} P_{n}$ where $P_{n}$ always denotes a polynomial of degree at most $n$.

TheOREM 2.6. Suppose $P_{n}$ is a polynomial of degree at most $n$ and $\left|w^{n} P_{n}(z)\right| \leq M$ for q.e. $z \in S_{w}$. Then:

(i) $\left|P_{n}(z)\right| \leq M \exp \left(n V_{\Sigma, Q}^{*}(z)\right)$ for all $z \in \mathbb{C}^{k}$;

(ii) $\left|w^{n} P_{n}(z)\right| \leq M \exp \left[n\left(V_{\Sigma, Q}^{*}(z)-Q(z)\right)\right]$ for all $z \in \Sigma$;

(iii) $\left|w^{n} P_{n}(z)\right| \leq M$ for q.e. $z \in \Sigma$.

In particular if we define the "sup" norm of a function $f$ on $K$ by

$$
\|f\|_{K}^{*}=\inf \left\{\|f\|_{K \backslash F}: F \text { is pluripolar, } F \subset K\right\}
$$

then Theorem 2.6(iii) may be restated as

$$
\left\|w^{n} P_{n}\right\|_{S_{w}}^{*}=\left\|w^{n} P_{n}\right\|_{\Sigma}^{*}, \quad n=1,2, \ldots
$$


Note that if $\Sigma$ is not pluripolar in a neighbourhood of any of its points and $f$ is continuous on $\Sigma$ then $\|f\|_{\Sigma}^{*}=\|f\|_{\Sigma}$.

ThEOREM 2.7. Let $S$ be any closed subset of $\Sigma$ such that $\left\|w^{n} P_{n}\right\|_{S}^{*}=$ $\left\|w^{n} P_{n}\right\|_{\Sigma}^{*}$ for all polynomials $P_{n}$. Then $S \supset S_{w}$.

TheOREM 2.8. Let $\Sigma$ be a closed subset of $\mathbb{C}^{k}$ that is not pluripolar in a neighbourhood of any of its points. Let $w$ be a continuous admissible weight on $\Sigma$. Then $\left\|w^{n} P_{n}\right\|_{\Sigma}=\left\|w^{n} P_{n}\right\|_{S_{w}}$ for all polynomials $P_{n}(n=1,2, \ldots)$. Furthermore, if $S$ is any closed subset of $\Sigma$ such that $\left\|w^{n} P_{n}\right\|_{\Sigma}=\left\|w^{n} P_{n}\right\|_{S}$ for all polynomials $P_{n}, n=1,2, \ldots$, then $S \supset S_{w}$.

In order to calculate the support $S_{w}$ of the extremal measure we utilize a characterization of the points in $S_{w}$. Before stating it, we need a definition.

DEFInITION 2.9. We say that the function $g$ attains its essential maximum modulus on the set $S$ in the subset $S_{1} \subset S$ if $\|g\|_{S \backslash S_{1}}^{*}<\|g\|_{S}^{*}$.

Proposition 2.10. Let $w$ be an admissible weight on $\Sigma$. Then $z \in \Sigma$ belongs to the support $S_{w}$ of the extremal measure $\mu_{w}$ if and only if for every neighbourhood $B$ of $z$ there exists a weighted polynomial $w^{n} P_{n}$ taking its essential maximum modulus on $\Sigma$ in $B \cap \Sigma$.

Proof. Let $z \in S_{w}$ and let $B$ be an arbitrary neighbourhood of $z$. By Theorem 2.7 applied to the closed set $\Sigma \backslash B$, there exists a weighted polynomial $w^{n} P_{n}$ with

$$
\left\|w^{n} P_{n}\right\|_{\Sigma \backslash B}^{*}<\left\|w^{n} P_{n}\right\|_{\Sigma}^{*}
$$

It follows that $w^{n} P_{n}$ takes its essential maximum modulus on $\Sigma$ in $\Sigma \cap B$. Conversely, if $w^{n} P_{n}$ takes its essential maximum modulus on $\Sigma$ in $\Sigma \cap B$, then $B \cap S_{w} \neq \emptyset$. Since the neighbourhood $B$ was arbitrary we conclude that $z \in S_{w}$.

Corollary 2.11. Suppose $w$ is a continuous admissible weight on $\Sigma$, and assume $\Sigma$ is not pluripolar in a neighbourhood of any of its points. Then $z \in S_{w}$ if and only if for every neighbourhood $B$ of $z$ there is a weighted polynomial $w^{n} P_{n}$ such that $w^{n} P_{n}$ attains its maximum modulus only in $B$.

Our next result deals with the behaviour of the weighted pluricomplex Green function under polynomial mappings and is a weighted version of a result by Klimek [3].

Theorem 2.12. Let $\alpha, \beta$ be positive integers and let $f: \mathbb{C}^{k} \rightarrow \mathbb{C}^{k}$ be a holomorphic mapping. Assume $f$ is a polynomial mapping of degree not greater than $\beta$ and

$$
\liminf _{|z| \rightarrow \infty} \frac{|f(z)|}{|z|^{\alpha}}>0
$$


Then $f$ is proper and for every $E \subset \mathbb{C}^{k}$ with weights $\widetilde{w}$ on $f^{-1}(E)$ and $w$ on $E$ such that $\widetilde{w}=w \circ f$ we have

$$
\alpha V_{f^{-1}(E), \widetilde{Q} / \alpha} \leq V_{E, Q} \circ f \leq \beta V_{f^{-1}(E), \widetilde{Q} / \beta} .
$$

Proof. By our first inequality $f$ is proper and proper polynomial mappings are surjective. Take a set $E \subset \mathbb{C}^{k}$ and a function $u \in \mathcal{L}$ such that $\alpha u \leq \widetilde{Q}:=Q \circ f$ on $f^{-1}(E)$. The function

$$
v(z):=\alpha \sup u\left(f^{-1}(z)\right)=\alpha \sup \left\{u(x): x \in f^{-1}(z)\right\}
$$

is plurisubharmonic by Proposition 2.9.26 in [4]. Moreover, suppose $M, N$ are chosen such that

$$
\limsup _{|z| \rightarrow \infty}\left(u(z)-\log ^{+}|z|\right)<M \quad \text { and } \quad \liminf _{|z| \rightarrow \infty} \frac{|f(z)|}{|z|^{\alpha}}>N .
$$

Then we have

$$
\begin{aligned}
\limsup _{|z| \rightarrow \infty}\left(v(z)-\log ^{+}|z|\right)= & \limsup _{|x| \rightarrow \infty}\left(\alpha \sup u\left(f^{-1}(f(x))\right)-\log ^{+}|f(x)|\right) \\
= & \limsup _{|x| \rightarrow \infty}\left[\alpha \sup u\left(f^{-1}(f(x))\right)-\alpha \log ^{+}|x|\right. \\
& \left.+\alpha \log ^{+}|x|-\log ^{+}|f(x)|\right] \\
\leq & \alpha M-\log N<\infty
\end{aligned}
$$

implying $v \in \mathcal{L}$. Since $\alpha u \leq \widetilde{Q}=Q \circ f$ on $f^{-1}(E)$ the function $v$ satisfies

$$
v(z)=\alpha \sup u\left(f^{-1}(z)\right) \leq \alpha\left(\frac{1}{\alpha} \widetilde{Q} \circ f^{-1}(z)\right) \quad \text { on } E .
$$

It follows that

$$
\alpha u(x) \leq v(f(x)) \leq\left(V_{E, Q} \circ f\right)(x) \quad \text { for any } x \in \mathbb{C}^{k} .
$$

Thus we have

$$
V_{f^{-1}(E), \widetilde{Q} / \alpha} \leq \frac{1}{\alpha} V_{E, Q} \circ f .
$$

To establish our second inequality note that $\operatorname{deg} f \leq \beta$ implies

$$
\limsup _{|z| \rightarrow \infty} \frac{|f(z)|}{|z|^{\beta}}<\infty
$$

Clearly if $u \in \mathcal{L}$ then $\frac{1}{\beta}(u \circ f) \in \mathcal{L}$. Furthermore, if $u \leq Q$ on $E$ we have

$$
\frac{1}{\beta} u \circ f \leq \frac{1}{\beta} Q \circ f=\frac{1}{\beta} \widetilde{Q} \quad \text { on } f^{-1}(E) .
$$

Taking the supremum over all such functions completes the argument.

Corollary 2.13. Let $\alpha$ be a positive integer and let $f: \mathbb{C}^{k} \rightarrow \mathbb{C}^{k}$ be a holomorphic mapping. Assume $f$ is a polynomial mapping of degree not 
greater than $\alpha$ and

$$
\liminf _{|z| \rightarrow \infty} \frac{|f(z)|}{|z|^{\alpha}}>0 .
$$

Then $f$ is proper and for every $E \subset \mathbb{C}^{k}$ with weight $w$ on $E$ and $\widetilde{w}=w \circ f$ on $f^{-1}(E)$ we have

$$
V_{E, Q} \circ f=\alpha V_{f^{-1}(E), \widetilde{Q} / \alpha} .
$$

As a special case, Theorem 2.12 includes Theorem 1.10(f), found on page 199 of [9]. Corollary 2.13 will be used in Section 3 to construct a useful example. It will also be necessary in Section 3 to utilize the following lemma, provided by Bloom.

Lemma 2.14. Let $K \subset \mathbb{C}^{k}$ be compact and $w$ a continuous admissible weight on $K$. Then for some $\varepsilon>0$ we have $w \geq \varepsilon$ on $S_{w}$.

Proof. Let $K_{j}:=\{z \in K: w \geq 1 / j\}$ for $j \in \mathbb{N}$. We will show that for $j$ sufficiently large, $V_{K_{j}, Q}^{*}=V_{K, Q}^{*}$. Then we will have $\left(d d^{c} V_{K, Q}^{*}\right)^{k}=$ $\left(d d^{c} V_{K_{j}, Q}^{*}\right)^{k}$ and it follows that $w \geq 1 / j$ on $S_{w}$.

By assumption $w$ is admissible so the set $\{z \in K: w(z)>0\}$ is not pluripolar. Observe that $\{z \in K: w(z)>0\}=\bigcup_{j=1}^{\infty} K_{j}$. For some $j_{0}$ the set $K_{j_{0}}$ is not pluripolar. There is a constant $c>0$ such that $V_{K_{j_{0}, Q}}(z)<c$ for all $z \in K$. Choose $j_{1} \geq j_{0}$ so that $Q(z) \geq c$ on $K \backslash K_{j_{1}}$; it suffices to choose $j_{1}$ so that $1 / j_{1}<c$. Let $u \in \mathcal{L}$ and $u(z) \leq Q(z)$ on $K_{j_{1}}$. Then

$$
u(z) \leq V_{K_{j_{1}}, Q}^{*}(z) \leq V_{K_{j_{0}}, Q}^{*}(z) \leq c
$$

for all $z \in K$, implying that $u \leq Q$ on $K \backslash K_{j_{1}}$. It follows that $u \leq Q$ on $K$ and thus $V_{K_{j}, Q}^{*} \leq V_{K, Q}$. Finally, the fact that $K_{j_{1}} \subset K$ immediately gives the reverse inequality. Hence $V_{K_{j_{1}}, Q}^{*}=V_{K, Q}^{*}$.

The unweighted versions of the following two results are Corollary 5.2.5 and 5.2.6 in [4]. Our next result is Proposition 3.11 in [11].

Proposition 2.15. Suppose that $E \subset \mathbb{C}^{k}$ is bounded and $F \subset \mathbb{C}^{k}$ is pluripolar. Let $w$ be an admissible continuous weight on $E \cup F$. Then $V_{E \cup F, Q}^{*}=V_{E, Q}^{*}$.

Proof. It is clear that $V_{E \cup F, Q}^{*} \leq V_{E, Q}^{*}$ so we simply have to prove that

$$
V_{E, Q}^{*} \leq V_{E \cup F, Q}^{*} \text {. }
$$

Let $u \in \mathcal{L}$ be such that $u \leq Q$ on $E$ and take $v \in \mathcal{L}$ such that $v=-\infty$ on $F$. This is possible by Theorem 5.2.4 in [4] which states a pluripolar set $F$ is $\mathcal{L}$-polar. We may assume $v \leq 0$ on $E$. Then for each $\varepsilon>0$,

$$
u+\varepsilon v \leq V_{E \cup F, Q} \leq V_{E \cup F, Q}^{*} .
$$


Therefore $u \leq V_{E \cup F, Q}^{*}$ quasi-everywhere, and hence everywhere on $\mathbb{C}^{k}$. It follows that $V_{E, Q}^{*} \leq V_{E \cup F, Q}^{*}$ as required.

Proposition 2.16. Let $\left\{E_{j}\right\}_{j \in \mathbb{N}}$ be an increasing sequence of sets in $\mathbb{C}^{k}$, whose union $E$ is bounded. Let $w$ be an admissible weight on $E$. Then

$$
\lim _{j \rightarrow \infty} V_{E_{j}, Q}^{*}=V_{E, Q}^{*} .
$$

Proof. We assume without loss of generality that $E_{1}$ is not pluripolar. Since negligible sets are pluripolar, the set $\left\{z \in E_{j}: V_{E_{j}, Q}^{*}>Q\right\}$ is pluripolar for every $j$. Furthermore, the countable union of pluripolar sets is pluripolar, implying that

$$
F=\bigcup_{j \in \mathbb{N}}\left\{z \in E_{j}: V_{E_{j}, Q}^{*}>Q\right\}
$$

is pluripolar. Define $V=\lim _{j \rightarrow \infty} V_{E_{j}, Q}^{*}$. It is clear that $V \in \mathcal{L}$ and $V=$ $Q$ on $E \backslash F$. It follows that $V \leq V_{E \backslash F, Q}^{*}$. By the previous proposition, $V_{E, Q}^{*}=$ $V_{E \backslash F, Q}^{*}$ so our inequality becomes $V \leq V_{E, Q}^{*}$. Finally, the fact $E=\bigcup E_{j}$ yields $V \geq V_{E, Q}^{*}$.

One final result concerning weighted pluripotential theory is required. In general, the weighted pluricomplex Green function can be expressed as the upper envelope of functions of the form $[\operatorname{deg}(P)]^{-1} \log |P|$, where $P$ is a complex-valued polynomial, that is, $P \in \mathbb{C}\left[z_{1}, \ldots, z_{k}\right]=\mathbb{C}[z]$. This is proven as Theorem 2.8 of Appendix B of [9]. For the remainder of this paper we restrict our attention to closed subsets of $\mathbb{R}^{k}$, so it is natural to require our polynomials to have real coefficients.

Lemma 2.17. For closed sets $\Sigma \subset \mathbb{R}^{k}$ with an admissible weight $w$,

$$
V_{\Sigma, Q}(z)=\lim _{n \rightarrow \infty} \sup \left\{\frac{1}{n} \log \left|P_{n}(z)\right|: P_{n} \in \mathbb{R}[z] \text { and }\left\|w^{n} P_{n}\right\|_{\Sigma} \leq 1\right\}
$$

where we restrict to the class $\mathbb{R}[z]$ of polynomials with real coefficients.

Proof. Let $\Sigma$ be a closed set in $\mathbb{R}^{k}$ and take $w$ to be an admissible weight on $\Sigma$. Let $\mathcal{F}_{n}:=\left\{P_{n} \in \mathbb{C}[z]:\left\|w^{n} P_{n}\right\|_{\Sigma} \leq 1\right\}$. Define $\phi_{n}(z)=$ $\sup _{P_{n} \in \mathcal{F}_{n}}\left|P_{n}(z)\right|$. Then from Appendix B of [9] we have

$$
V_{\Sigma, Q}(z)=\lim _{n \rightarrow \infty} \frac{1}{n} \log \phi_{n}(z) .
$$

Analogously, let $\mathcal{G}_{n}=\left\{R_{n} \in \mathbb{R}[z]:\left\|w^{n} R_{n}\right\|_{\Sigma} \leq 1\right\}$ where $R_{n}$ denotes a polynomial with real coefficients and of degree at most $n$. Taking $\psi_{n}(z)=$ $\sup _{R_{n} \in \mathcal{G}_{n}}\left|R_{n}(z)\right|$ we have a well-defined function

$$
V_{\mathbb{R}}(z)=\lim _{n \rightarrow \infty} \frac{1}{n} \log \psi_{n}(z) .
$$

We have $\mathcal{G}_{n} \subset \mathcal{F}_{n}$, and consequently $\psi_{n} \leq \phi_{n}$. Hence $V_{\mathbb{R}}(z) \leq V_{\Sigma, Q}(z)$ on $\mathbb{C}^{k}$. 
Observe that a complex multivariable polynomial $P_{n}(z)=\sum_{|j| \leq n} \alpha_{j} z^{j}$ may be decomposed by taking the real and imaginary parts of $\alpha_{j}$. That is, $P_{n}(z)=A(z)+i B(z)$ where $A(z)=\sum_{|j| \leq n} \Re\left(\alpha_{j}\right) z^{j}$ and $B(z)=\sum_{|j| \leq n} \Im\left(\alpha_{j}\right) z^{j}$. For $x \in \mathbb{R}^{k}$ this gives a decomposition of $P_{n}(x)$ into real and imaginary parts with $|A(x)| \leq|P(x)|$ and $|B(x)| \leq|P(x)|$. Given $P_{n} \in \mathcal{F}_{n}$ the corresponding polynomials $A, B$ are elements of $\mathcal{G}_{n}$. Therefore, fixing $z \in \mathbb{C}^{k}$ we have

$$
|P(z)| \leq|A(z)|+|B(z)| \leq 2 \psi_{n}(z) .
$$

Taking the supremum over $\mathcal{F}_{n}$ yields $\phi_{n}(z) \leq 2 \psi_{n}(z)$. It follows that

$$
\lim _{n \rightarrow \infty} \frac{1}{n} \log \phi_{n}(z) \leq \lim _{n \rightarrow \infty} \frac{1}{n} \log \psi_{n}(z)
$$

and thus $V_{\Sigma, Q}(z) \leq V_{\mathbb{R}}(z)$.

3. The approximation problem. This section is focused on answering the following question:

Given a closed set $\Sigma \subset \mathbb{R}^{k} \subset \mathbb{C}^{k}, k>1$, and a continuous admissible weight $w$ on $\Sigma$, when will a continuous function $f$ on $\Sigma$ be uniformly approximable by a sequence $\left\{w^{n} P_{n}\right\}_{n=1}^{\infty}$ of weighted polynomials?

We will refer to this as the weighted approximation problem. For a given set $\Sigma$ with continuous weight $w$ we consider the weighted approximation problem solved if we identify the set $Z(w)$ as defined in Theorem 3.1.

For the remainder of this paper, $P_{n}$ denotes a real-valued polynomial with degree at most $n$. This weighted approximation problem was considered on the real line by Kuijlaars, Mhaskar and Saff, and Totik. Their results were preceded by many specialized results for individual weights, starting with Lorentz' incomplete polynomials on $[0,1]$. The first result is a StoneWeierstrass type theorem, proven using the same technique employed in the one-variable setting.

ThEOREM 3.1. Let $\Sigma \subset \mathbb{R}^{k}$ be a closed set and $w$ a continuous, admissible weight on $\Sigma$. Then there exists a closed set $Z=Z(w) \subset \Sigma$ such that a continuous function $f$ on $\Sigma$ is the uniform limit of weighted polynomials $\left\{w^{n} P_{n}\right\}_{n=1}^{\infty}$ if and only if $f$ vanishes on $Z$.

Proof. To establish our result, we use the Stone-Weierstrass theorem, stated below.

Let $C(X)$ denote the family of real-valued continuous functions on a compact Hausdorff space $X$ and for $\mathcal{A} \subset C(X)$ let $Z(\mathcal{A})$ denote the set of points $x \in X$ such that $f(x)=0$ for every $f \in \mathcal{A}$. Suppose that $\mathcal{A}$ has the following properties;

(a) If $f, g \in \mathcal{A}$ then $\alpha f+\beta g \in \mathcal{A}$ for all real $\alpha$ and $\beta$.

(b) If $f, g \in \mathcal{A}$ then $f g \in \mathcal{A}$. 
(c) $\mathcal{A}$ is closed under uniform limits.

(d) If $x, y \in X \backslash Z(\mathcal{A})$ then there is an $f \in \mathcal{A}$ such that $f(x) \neq f(y)$.

Then $\mathcal{A}=\{f \in C(X): f \equiv 0$ on $Z(\mathcal{A})\}$.

In other words, we need to show that $\mathcal{A}$ is a closed algebra which separates points in $X \backslash Z(\mathcal{A})$.

From Theorem 2.6(ii) we have

$$
\left|w^{n} P_{n}(x)\right| \leq\left\|w^{n} P_{n}\right\|_{S_{w}} \exp \left[n\left(V_{\Sigma, Q}^{*}(x)-Q(x)\right)\right] \quad \text { for all } x \in \Sigma .
$$

Since $V_{\Sigma, Q}^{*} \in \mathcal{L}$, for some constant $\kappa>0$ we have

$$
V_{\Sigma, Q}^{*}(z) \leq \log ^{+}|z|+\kappa .
$$

Let $X=\{x \in \Sigma:|x| \leq \varrho\}$ where $\varrho$ is a sufficiently large constant so that

$$
Q(x)-\log |z| \geq \kappa+1 \quad \text { for } x \notin X .
$$

Observe that for $x \notin X$,

$$
\begin{aligned}
\left|w^{n} P_{n}(x)\right| & \leq\left\|w^{n} P_{n}\right\|_{S_{w}} \exp \left[n\left(\log ^{+}|x|+\kappa-\log |x|-\kappa-1\right)\right] \\
& =\left\|w^{n} P_{n}\right\|_{S_{w}} \exp [-n] .
\end{aligned}
$$

Hence the uniform convergence of any sequence $\left\{w^{n} P_{n}\right\}$ on $\Sigma$ is equivalent to its uniform convergence on $X$.

Now let $\mathcal{A}$ be the collection of all continuous functions $f$ on $X$ such that $f$ is the uniform limit of a sequence $\left\{w^{n} P_{n}\right\}_{n=1}^{\infty}$ of weighted polynomials. Our collection $\mathcal{A}$ satisfies condition (a) since if $w^{n} P_{n} \rightarrow f$ and $w^{n} R_{n} \rightarrow g$ uniformly on $X$ then $w^{n}\left(\alpha P_{n}+\beta R_{n}\right) \rightarrow \alpha f+\beta g$ uniformly on $X$. To see that condition (b) is satisfied, let $T_{2 n}=P_{n} R_{n}, T_{2 n+1}=P_{n+1} R_{n}$. Then the sequence $\left\{w^{n} T_{n}\right\}$ tends uniformly to $f g$ on $X$. A standard diagonal argument proves that condition (c) is met.

To show that (d) holds, observe that for $\widetilde{x} \in X \backslash Z(\mathcal{A})$ there is a function $g \in \mathcal{A}$ such that $g(\widetilde{x}) \neq 0$. Note that $w(x)>0$ for $x \in X \backslash Z(\mathcal{A})$. Suppose that $w^{n} P_{n} \rightarrow g$ uniformly on $X$. Given $y \in X \backslash Z(\mathcal{A})$ such that $y \neq \widetilde{x}$, there exists $j \in\{1, \ldots, k\}$ such that $\widetilde{x}_{j} \neq y_{j}$. Assume without loss of generality that $j=1$. Taking $R_{n+1}(x)=\left(x_{1}-y_{1}\right) P_{n}(x)$ and $f(x)=w(x)\left(x_{1}-y_{1}\right) g(x)$ we see that $w^{n+1} R_{n+1}$ converges uniformly to $f$, hence $f \in \mathcal{A}$. By construction $f(\widetilde{x}) \neq f(y)$, thus $\mathcal{A}$ meets all of the conditions of the Stone-Weierstrass theorem. Therefore we apply the Stone-Weierstrass theorem to conclude that there exists a closed set $Z(w)$ such that a continuous function $f$ will be the uniform limit of weighted polynomials iff $f$ vanishes on $Z(w)$.

TheOREM 3.2. Suppose we have a closed set $\Sigma \subset \mathbb{R}^{k}$, not pluripolar in a neighbourhood of any of its points, with a continuous admissible weight function $w$. If a sequence $\left\{w^{n} P_{n}\right\}_{n=1}^{\infty}$ of weighted polynomials converges uniformly on $S_{w}$ then $\left\{w^{n}(\gamma) P_{n}(\gamma)\right\}$ tends to zero for every $\gamma \in \Sigma \backslash S_{w}$. 
Proof. Assume to the contrary that $w^{n} P_{n}$ converges to some $g$ uniformly on $S_{w}$ but $w^{n}(\gamma) P_{n}(\gamma)$ does not tend to zero for some $\gamma \in \Sigma \backslash S_{w}$. Let $\mathcal{A}$ be the collection of all continuous functions $f$ on $S_{w}$ that are uniform limits of weighted polynomials $w^{n} R_{n}$, with the additional property that $R_{n}(\gamma)=0$. It is clear that $\mathcal{A}$ satisfies the assumptions of the Stone-Weierstrass theorem, hence there exists a set $Z \subset S_{w}$ such that $f \in \mathcal{A}$ if and only if $f$ vanishes on $Z$. Observe that from Lemma 2.14 we have $w(z)>0$ for $z \in S_{w}$.

We claim that $g \in \mathcal{A}$. Clearly,

$$
w(x)^{n+2}|x-\gamma|^{2} P_{n}(x) \rightarrow w(x)^{2}|x-\gamma|^{2} g(x) \quad \text { on } S_{w} .
$$

This implies that $w(x)^{2}|x-\gamma|^{2} g(x) \in \mathcal{A}$ and so $w(x)^{2}|x-\gamma|^{2} g(x)$ must vanish on $Z$. Furthermore, $g$ vanishes on $S_{w}$ precisely where $w(x)^{2}|x-\gamma|^{2} g(x)$ does, thus $g \in \mathcal{A}$. Therefore, there are polynomials $R_{n}$ with $R_{n}(\gamma)=0$ such that $w^{n} R_{n} \rightarrow g$ on $S_{w}$. It follows that $w^{n}\left(P_{n}-R_{n}\right)(\gamma)$ converges uniformly to zero on $S_{w}$. But $w^{n} P_{n}(\gamma)$ does not tend to zero, hence there is an $\varepsilon>0$ and an integer $N$ such that

$$
\left\|w^{N}\left(P_{N}-R_{N}\right)\right\|_{S_{w}} \leq \varepsilon \quad \text { while } \quad\left|w^{N}\left(P_{N}-R_{N}\right)(\gamma)\right|>\varepsilon
$$

But this contradicts Theorem 2.8 which states that

$$
\left\|w^{N}\left(P_{N}-R_{N}\right)\right\|_{\Sigma}=\left\|w^{N}\left(P_{N}-R_{N}\right)\right\|_{S_{w}} .
$$

For $\Sigma \subset \mathbb{R}$, Theorems 3.1 and 3.2 were proven on the real line by Kuijlaars in [6]. His proofs also depended on the Stone-Weierstrass theorem. A weaker version of Theorem 3.2 was proven by Totik in [12]. We will now construct a useful example using Corollary 2.13.

EXAMPLE 3.3. Let $w(t)=|t|^{\theta /(1-\theta)}$ with $0<\theta<1$ on $\Sigma=[-1,1] \subset$ $\mathbb{R} \subset \mathbb{C}$. We will calculate the support of $\mu_{w}$ and the set $Z(w)$. The first step is to calculate $V_{\Sigma, Q}$ using Corollary 2.13. From [10] we know that for $E=[0,1]$ with weight $v(t)=t^{\theta /(1-\theta)}, 0<\theta<1$, the weighted Green function is

$$
V_{E, Q_{v}}(t)=\log |\phi(t)|-\frac{\theta}{1-\theta}\left(\log t-\log \left|\frac{\phi(t)-\phi(0)}{\phi(0) \phi(t)-1}\right|-\log |\phi(t)|\right)
$$

where $\phi$ is the mapping

$$
\phi(t)=\frac{\sqrt{t-\theta^{2}}+\sqrt{t-1}}{\sqrt{t-\theta^{2}}-\sqrt{t-1}} .
$$

Observe that for $f(t)=t^{2}$ we have $f^{-1}(E)=[-1,1]$. Therefore, by Corollary 2.13 ,

$$
\begin{aligned}
V_{\Sigma, Q_{w}}(t)= & \frac{1}{2} \log \left|\phi\left(t^{2}\right)\right|-\frac{\theta}{1-\theta} \log |t| \\
& +\frac{\theta}{2(1-\theta)}\left(\log \left|\frac{\phi\left(t^{2}\right)-\phi(0)}{\phi(0) \phi\left(t^{2}\right)-1}\right|-\log \left|\phi\left(t^{2}\right)\right|\right) .
\end{aligned}
$$


Furthermore, it was also calculated that the support of the extremal measure $\mu_{v}$ on $E$ is $\left[\theta^{2}, 1\right]$. It follows that the support of the extremal measure $\mu_{w}$ on $\Sigma$ is $[-1,-\theta] \cup[\theta, 1]$. Finally, since $Q_{w}$ is convex we may apply Theorem 4.3 from [12] to conclude that $Z(w)=[-\theta, \theta]$.

Note: Replacing $\theta /(1-\theta)$ by $\alpha$, we have $w(t)=|t|^{\alpha}$ on $\Sigma=[-1,1]$ where $\alpha>0$. Then the support of the extremal measure is $S_{w}=\left[-1,-\frac{\alpha}{1+\alpha}\right] \cup$ $\left[\frac{\alpha}{1+\alpha}, 1\right]$ and $Z(w)=\left[-\frac{\alpha}{1+\alpha}, \frac{\alpha}{1+\alpha}\right]$. We tend to use $\theta /(1-\theta)$ since we are interested in applications to the theory of $\theta$-incomplete polynomials.

THEOREM 3.4. Suppose we have a continuous even admissible weight $v(t)$ on the interval $[-1,1]$. Then for the weight $w(x)=v(|x|)$ on the real unit ball $\Sigma=\left\{x \in \mathbb{R}^{k}:|x| \leq 1\right\}$ we have $Z(w)=\{x \in \Sigma:|x| \in Z(v)\}$.

Proof. Suppose there exists a point $q \in\{x \in \Sigma:|x| \in Z(v)\}$ and a continuous function $f$ on $\Sigma$ which can be uniformly approximated by weighted polynomials $w^{n} P_{n}$ but is nonzero at $q$. Consider the line passing through the point $q$ and the origin, $L=\left\{t x^{*}:-1 \leq t \leq 1\right\}$ for some $x^{*} \in \partial \Sigma$. Observe that for points on $L$ our weight becomes

$$
w\left(t x^{*}\right)=v\left(\left|t x^{*}\right|\right)=v(|t|)
$$

since $x^{*}$ is a boundary point of $\Sigma$. Restricting our sequence $\left\{w^{n} P_{n}\right\}_{n=1}^{\infty}$ to $L$ gives rise to a sequence of weighted polynomials in variable $t$ on $[-1,1]$ for the weight $v(t)$. By definition, this sequence tends to zero for every $t \in Z(v)$. However, the uniform limit of this sequence is the function $h(t)=f\left(t x^{*}\right)$ and $h$ is nonzero at $\bar{t} \in Z(v)$ where $q=\bar{t} x^{*}$. This is impossible, and consequently our original sequence on $\Sigma$ could not have $f$ as its uniform limit if $f(q) \neq 0$. Hence, we have $\{x \in \Sigma:|x| \in Z(v)\} \subset Z(w)$.

To establish the reverse inclusion we will prove the contrapositive. Take a point $q \in \Sigma$ such that $r=|q| \notin Z(v)$. Let $f$ be a continuous even function on $[-1,1]$, nonzero at $r$ and vanishing on $Z(v)$. By the definition of $Z(v)$ our function $f$ is uniformly approximable by a sequence $\left\{v(t)^{n} P_{n}(t)\right\}_{n=1}^{\infty}$. Since $f$ is an even function we have

$$
v(t)^{n}\left(\frac{P_{n}(t)+P_{n}(-t)}{2}\right) \rightarrow f \quad \text { uniformly. }
$$

Consequently, we take our sequence to be of the form $\left\{v\left(t^{2}\right)^{n / 2} R_{n / 2}\left(t^{2}\right)\right\}_{n=1}^{\infty}$. Let $t=|x|$. Under this substitution, our weight $v(t)$ becomes the weight $w(x)$ on $\Sigma$. We let $T_{n}\left(x_{1}, \ldots, x_{k}\right)=R_{n / 2}\left(x_{1}^{2}+\cdots+x_{k}^{2}\right)$ so that $w^{n} T_{n} \rightarrow$ $h\left(x_{1}, \ldots, x_{k}\right)$ uniformly, where $h\left(x_{1}, \ldots, x_{k}\right)=f(|x|)$ on $\Sigma$. By construction, $h(q) \neq 0$, and we have defined a sequence $w^{n} T_{n}, n=1,2, \ldots$, which uniformly converges to $h$ on $\Sigma$. Thus $q \notin Z(w)$. It follows that $Z(w) \subset\{x \in \Sigma$ : $|x| \in Z(v)\}$, allowing us to conclude that $Z(w)=\{x \in \Sigma:|x| \in Z(v)\}$. 
Corollary 3.5. Suppose we have a continuous even admissible weight $v(t)$ on the interval $[-1,1]$ and $S_{v}=[-1,1] \backslash \operatorname{int}(Z(v))$. Then for the weight $w(x)=v(|x|)$ on the real unit ball $\Sigma=\left\{x \in \mathbb{R}^{k}:|x| \leq 1\right\}$ we have $S_{w}=\Sigma \backslash \operatorname{int}(Z(w))$.

Proof. From Theorem 3.2 we know that $S_{w}$ must contain $\{x \in \Sigma$ : $|x| \notin Z(v)\}$. The support $S_{w}$ is a closed set and $S_{v}=[-1,1] \backslash \operatorname{int}(Z(v))$ so we have $\left\{x \in \Sigma:|x| \in S_{v}\right\} \subset S_{w}$. To show containment in the other direction, we argue by contradiction. Suppose there is a point $q \in S_{w}$ with $r=|q| \notin S_{v}$. Then $q \in \operatorname{int}(Z(w))$. Let $B$ denote a neighbourhood of $q$, and assume without loss of generality that $B$ is in the interior of $Z(w)$. Then, by Corollary 2.11, there exists a weighted polynomial $w^{n} P_{n}$ taking its maximum modulus on $\Sigma$ in $\Sigma \cap B$. Let $L$ be the line through $q$ and the origin, $L=\left\{t x^{*}:-1 \leq t \leq 1\right\}$ where $x^{*} \in \partial \Sigma$. Observe that $w^{n} P_{n}$, restricted to $L$, gives a univariate weighted polynomial $v(t)^{n} T_{n}(t)$ taking its maximum modulus on $B \cap L$, a neighbourhood of $r$. Consequently, $r$ should be in the support of the extremal measure for $L$, associated with the weight $v(t)$. But by assumption $r$ is not in $S_{v}$ since $S_{v} \cap \operatorname{int}(Z(w))=\emptyset$. It follows that $q \notin S_{w}$, yielding $S_{w}=\left\{x \in \Sigma:|x| \in S_{v}\right\}$.

We may now use Example 3.3 combined with Theorem 3.4 to solve the approximation problem for the real unit ball $\left\{x \in \mathbb{R}^{k}:|x| \leq 1\right\}$ with associated radial weight.

ExAmple 3.6. Let $\Sigma=\left\{x \in \mathbb{R}^{k}:|x| \leq 1\right\} \subset \mathbb{R}^{k} \subset \mathbb{C}^{k}$. Define a weight function on $\Sigma$ by $w(x)=|x|^{\theta /(1-\theta)}$ for $0<\theta<1$. Then we have $Z(w)=\theta \Sigma=\{x \in \Sigma:|x| \leq \theta\}$ and $S_{w}=\{x \in \Sigma: \theta \leq|x| \leq 1\}$.

Turning our attention to linear weights, we prove a theorem which will be extremely useful when considering multivariable incomplete polynomials. Let $S[a, b]$ denote the line segment with initial point $a$ and terminal point $b$ in $\mathbb{R}^{k}$, and let $M_{c}$ denote the ray through the point $c(c \neq 0)$ and the origin. Suppose $L$ is a hyperplane in $\mathbb{R}^{k}$ not containing the origin. Take a compact subset $\Gamma$ of $L$ together with all line segments from the origin to points of $\Gamma$. The resulting union $\Sigma:=\bigcup_{q \in \Gamma} S[0, q]$ will be called a compact section if it is not pluripolar in a neighbourhood of any of its points. If the equation of the hyperplane $L$ is $\sum_{j=1}^{k} c_{j} x_{j}=d$ then the associated linear form for $\Sigma$ is $\ell(x):=d^{-1} \sum_{j=1}^{k} c_{j} x_{j}$.

Given a compact set $\Sigma$, starlike with respect to the origin, and a point $p \in \Sigma$ we denote $\Sigma \cap M_{p}$ by $\mathcal{R}_{p}$. Recall $\Sigma$ is starlike with respect to the origin if for any point $q \in \Sigma$ the closed line segment joining the origin to $q$ lies entirely in $\Sigma$. If $\Sigma$ is a compact section then $\mathcal{R}_{p}$ for a given point $p \in \Sigma$ will be of the form $S[0, b]$ for some $b \in \Gamma$. 
TheOREM 3.7. Let $\Sigma$ be a compact section in $\mathbb{R}^{k}$ and suppose our weight function on $\Sigma$ is $w(x)=l(x)^{\theta /(1-\theta)}$ where $l$ denotes the associated linear form and $\theta$ is fixed, $0<\theta<1$. Then $Z(w)=\theta^{2} \Sigma=\left\{\theta^{2} x: x \in \Sigma\right\}$ and $S_{w}=\overline{\Sigma \backslash \theta^{2} \Sigma}$.

Proof. To show that $\theta^{2} \Sigma \subset Z$ we take a point $a \in \theta^{2} \Sigma$ and suppose $f$ is a continuous function on $\Sigma$ which is the uniform limit of weighted polynomials $\left\{w^{n} P_{n}\right\}_{n=1}^{\infty}$. Restrict to $\mathcal{R}_{a}=\{t b: 0 \leq t \leq 1\}$ with $a=t b$ for some $t \in\left[0, \theta^{2}\right]$ and $b \in \Gamma$. Then $\left.w^{n} P_{n}\right|_{\mathcal{R}_{a}}$ gives a sequence $t^{n \theta /(1-\theta)} T_{n}(t), n=$ $1,2, \ldots$, where $T_{n}(t)=P_{n}(t b)$. Then by the univariate result the sequence $\left\{t^{n \theta /(1-\theta)} T_{n}(t)\right\}$ tends to zero uniformly for $t \in\left[0, \theta^{2}\right]$, forcing our function $f$ on $\Sigma$ to be zero at $a$. Thus $\theta^{2} \Sigma \subset Z(w)$.

Conversely, suppose our point $a \in \Sigma$ lies outside of $\theta^{2} \Sigma$. We will produce a continuous function on $\Sigma$ which is the uniform limit of weighted polynomials and nonzero at $a$. Let $r=l(a)$ and take $g(t)$ to be a continuous function on $[0,1]$ which is the uniform limit of the sequence $\left\{t^{n \theta /(1-\theta)} P_{n}(t)\right\}_{n=1}^{\infty}$ and $g(r) \neq 0$. Then the substitution $t=l(x)$ gives a function $f$ on $\left\{x \in \mathbb{R}^{k}\right.$ : $0 \leq l(x) \leq 1\}$. Observe $f$ is nonzero at $a$ and $f$ is the uniform limit of the weighted polynomials $l(x)^{n \theta /(1-\theta)} P_{n}(l(x))$ where $P_{n}(l(x))$ is a polynomial of degree at most $n$. Hence, by restricting to $\Sigma$ we have shown that $Z(w)=\theta^{2} \Sigma$.

We turn our attention to $S_{w}$. By Theorem 3.2, $S_{w}$ is at least $\overline{\Sigma \backslash \theta^{2} \Sigma}$. To show that $S_{w}$ is precisely this set, take a point $q$ in its complement and let $B$ denote a neighbourhood of $q$, also contained in its complement. By Corollary 2.11, there exists a weighted polynomial $w^{m} P_{m}$, taking its maximum modulus on $\Sigma \cap B$. Restricting to $\mathcal{R}_{q}$ gives a weighted polynomial $t^{m \theta /(1-\theta)} Q_{m}(t)$. It follows that $l(q) \in S_{v}$ where $v(t)=t^{\theta /(1-\theta)}$ on $[0,1]$. This is impossible since $S_{v}=\left[\theta^{2}, 1\right]$, allowing us to conclude that $S_{w}=\overline{\Sigma \backslash \theta^{2} \Sigma}$.

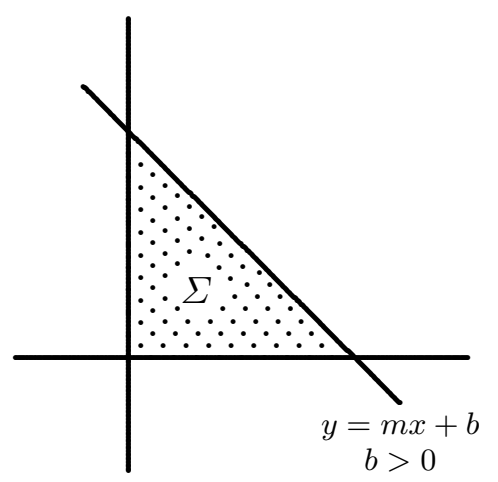

Fig. 1. Example of a compact section 
A simple example of a compact section is a $k$-simplex in $\mathbb{R}^{k}$ with a vertex at the origin. Note that compact sections are not necessarily convex, our definition only requires a compact section to be starlike with respect to the origin. It is helpful to explore a special case: we consider a 2 -simplex in $\mathbb{R}^{2}$ with a vertex at the origin.

EXAMPLE 3.8. Let $\Sigma$ be the closed triangle in $\mathbb{R}^{2}$ as in Figure 1. Let $l(x, y)=\frac{1}{b}(y-m x)$ and define our weight function $w$ on $\Sigma$ by

$$
w(x, y)=l(x, y)^{\theta /(1-\theta)} \quad \text { for a fixed } \theta, 0<\theta<1 .
$$

Then $Z(w)=\theta^{2} \Sigma$ and $S_{w}=\overline{\Sigma \backslash \theta^{2} \Sigma}$.

4. Approximation by incomplete polynomials. The weighted approximation problem discussed in the previous section evolved from the incomplete polynomials of G. G. Lorentz. We define a $\theta$-incomplete polynomial $P_{n}$ of degree $n$ in $\mathbb{R}^{k}, k \geq 1$, for a given $\theta, 0<\theta<1$, to be of the form

$$
P_{n}=\sum_{m \geq\lfloor\theta n\rfloor}^{n} H_{m}\left(x_{1}, \ldots, x_{k}\right)
$$

where $H_{m}$ is a polynomial, homogeneous of degree $m$ and $\lfloor\theta n\rfloor$ denotes the integer part of $\theta n$. We will always take $\theta$ to be in the open interval $(0,1)$.

On $[0,1]$ with weight $w(t)=t^{\theta /(1-\theta)}$ approximating by weighted polynomials is equivalent to approximating by $\theta$-incomplete polynomials. That is, given a continuous function $f$ on $[0,1]$, it is the uniform limit of $\theta$-incomplete polynomials if and only if it is uniformly approximable by weighted polynomials. We turn our attention to multivariable $\theta$-incomplete polynomials, beginning with a Stone-Weierstrass type theorem.

THEOREM 4.1. Given a compact set $\Sigma \subset \mathbb{R}^{k}$ and a real number $\theta$, $0<\theta<1$, there exists a closed set $Z_{\theta} \subset \Sigma$ such that a continuous function on $\Sigma$ is the uniform limit of a sequence $\left\{P_{n}\right\}_{n=1}^{\infty}$ of $\theta$-incomplete polynomials if and only if $f$ vanishes on $Z_{\theta}$.

We omit the proof of Theorem 4.1 since it is completely analogous to the proof of Theorem 3.1.

Proposition 4.2. Let $\Sigma$ be a compact set in $\mathbb{R}^{k}$, starlike with respect to the origin. Then in order for a continuous function $f$ on $\Sigma$ to be the uniform limit of a sequence $\left\{P_{n_{i}}\right\}_{i=1}^{\infty}$ of $\theta$-incomplete polynomials for a fixed $\theta, f$ must vanish on $\theta^{2} \Sigma$.

Proof. Suppose $f$ is the uniform limit of $\theta$-incomplete polynomials $\left\{P_{n_{i}}\right\}_{i=1}^{\infty}$ on $\Sigma$. Take a closed line segment $S[0, b]$ in $\Sigma$ for a boundary 
point $b$. Then $S[0, b]=\{t b: 0 \leq t \leq 1\}$. Restricting to $S[0, b]$ gives a sequence of univariate $\theta$-incomplete polynomials $R_{n_{i}}(t)=P_{n_{i}}(t b)$ converging uniformly to the continuous function $g(t)=f(t b)$ on $[0,1]$. Therefore, by the combined results of Lorentz and Kuijlaars, $R_{n_{i}}$ tends to zero as $n_{i} \rightarrow \infty$ for $t \in\left[0, \theta^{2}\right]$. It follows that $f \equiv 0$ on $\theta^{2} \Sigma$.

Corollary 4.3. Let $\Sigma$ be a compact set in $\mathbb{R}^{k}$, starlike with respect to the origin. Then $\theta^{2} \Sigma \subset Z_{\theta}$ for $Z_{\theta}$ as defined in Theorem 4.1 .

The proof of Proposition 4.2 is very similar to the proof of Proposition 1 in [5]. In the same paper, Kroó considered incomplete polynomials on a restricted class of convex domains which included sections of the unit disk with central angle less than $\pi / 2$ and parallelograms with a vertex at the origin in $\mathbb{R}^{2}$. Our next result shows that on compact sections, approximation with $\theta$-incomplete polynomials is equivalent to approximating with weighted polynomials for the weight used in Theorem 3.8.

TheOREM 4.4. Let $\Sigma$ be a compact section in $\mathbb{R}^{k}$ and $\theta$ be fixed. Then a continuous function $f$ on $\Sigma$ is the uniform limit of a sequence $\left\{P_{n}\right\}_{n=1}^{\infty}$ of $\theta$-incomplete polynomials if and only if $f \equiv 0$ on $\theta^{2} \Sigma$. In other words, $Z_{\theta}=\theta^{2} \Sigma$.

Proof. By definition, $\Sigma$ is a compact set in $\mathbb{R}^{k}$, starlike with respect to the origin. By Corollary $4.3, Z_{\theta}$ is at least $\theta^{2} \Sigma$. Conversely, suppose we have a continuous function $f$ on $\Sigma$ and $f \equiv 0$ on $\theta^{2} \Sigma$. Then by Theorem 3.7, the function $f$ is the uniform limit of a sequence $\left\{\ell(x)^{n \theta /(1-\theta)} P_{n}(x)\right\}_{n=1}^{\infty}$ of weighted polynomials where $\ell$ is the linear form associated to $\Sigma$. Actually from the proof of Theorem 3.7 we may write our sequence as $\left\{\ell(x)^{n \theta /(1-\theta)}\right.$ - $\left.P_{n}(\ell(x))\right\}_{n=1}^{\infty}$. We will use this sequence of weighted polynomials to construct a sequence of $\theta$-incomplete polynomials also uniformly approximating $f$. Observe that $\ell(x)^{n \theta /(1-\theta)} P_{n}(\ell(x))$ has degree at most $n+n \theta /(1-\theta)$ and $\theta(n+n \theta /(1-\theta))=\theta n /(1-\theta)$.

We add an additional assumption for the moment, namely assume $\theta$ is rational. Then we may write $\theta /(1-\theta)=p / q$ where $p, q \in \mathbb{N}$. For $n=m q$ where $m=1,2, \ldots$, we have $n \theta /(1-\theta)=m p$ and $\ell(x)^{m p} P_{m q}(\ell(x))$ is a $\theta$-incomplete polynomial. Therefore from the original sequence of weighted polynomials we have a subsequence $\left\{\ell(x)^{m p} P_{m q}(\ell(x))\right\}_{m=1}^{\infty}$ of $\theta$-incomplete polynomials whose uniform limit is $f$. We need a sequence $\left\{R_{n}(x)\right\}_{n=1}^{\infty}$ uniformly converging to $f$. Let $P_{m q}(\ell(x))=: P_{m q, 0}(x)$. Observe that the continuous functions $f(x) / \ell(x)^{j}$ for $j=1, \ldots, p+q-1$ also vanish on $\theta^{2} \Sigma$. Therefore there exist corresponding sequences $\left\{\ell(x)^{m p} P_{m q, j}(x)\right\}_{m=1}^{\infty}$ whose uniform limit is $f / \ell^{j}$ for $j=1, \ldots, p+q-1$. It follows that the sequences $\left\{\ell(x)^{m p+j} P_{m q, j}(x)\right\}_{m=1}^{\infty}$ uniformly converge to $f$. Since for every natural number $n \geq p+q$ there is a pair $\{j, m\}$ such that $n=m(p+q)+j$, we 
have constructed a sequence $\left\{R_{n}(x)\right\}_{n=p+q}^{\infty}$ uniformly converging to $f$. Define $R_{n}(x)=\ell(x)^{n}$ for $n=1, \ldots, p+q-1$ in order to get a sequence $\left\{R_{n}(x)\right\}_{n=1}^{\infty}$. Hence for rational $\theta$ we have $Z_{\theta}=\theta^{2} \Sigma$.

Now for an arbitrary $\theta$, take $\theta_{1}$ to be rational and satisfying $\theta<\theta_{1}<1$. By the previous argument $Z_{\theta_{1}}=\theta_{1}^{2} \Sigma$. Given any continuous function $f \equiv 0$ on $\theta^{2} \Sigma$ we may define the continuous function $g$ on $\Sigma$ by

$$
g= \begin{cases}0 & \text { if } z \in \theta_{1}^{2} \Sigma \\ f & \text { if } z \in \Sigma \backslash \theta_{1}^{2} \Sigma .\end{cases}
$$

Then $g$ is the uniform limit of $\theta_{1}$-incomplete polynomials $\left\{P_{n}(x)\right\}_{n=1}^{\infty}$. Moreover, since $\theta_{1}>\theta$ each $\theta_{1}$-incomplete polynomial is also a $\theta$-incomplete polynomial. Therefore given any rational $\theta_{1}>\theta$ we have $Z_{\theta} \subset Z_{\theta_{1}}=\theta_{1}^{2} \Sigma$. It follows that $Z_{\theta}=\theta^{2} \Sigma$.

Note that in $\mathbb{R}^{2}$ this includes Lemma 3 of [5] which was proven using approximation theory. Our goal is to find a class of sets $\Sigma$, more general than compact sections, for which $Z_{\theta}$ will also be $\theta^{2} \Sigma$.

THEOREM 4.5. Let $\Sigma$ be the arbitrary intersection of compact sections in $\mathbb{R}^{k}$. Then for a fixed $\theta$ we have $Z_{\theta}=\theta^{2} \Sigma$.

Proof. Fixing $\theta$ we know from Corollary 4.3 that $Z_{\theta}$ is at least $\theta^{2} \Sigma$. In order to establish equality we show that given a point $x_{0} \in \Sigma \backslash \theta^{2} \Sigma$ it is possible to produce a continuous function $f$ on $\Sigma$ which is uniformly approximable by $\theta$-incomplete polynomials $\left\{P_{n}(x)\right\}_{n=1}^{\infty}$ and nonzero at $x_{0}$. Given such a point $x_{0}$, we know that for some compact section $\Delta_{i}$ we have $\Sigma \subset \Delta_{i}$ and $x_{0} \in \Delta_{i} \backslash \theta^{2} \Delta_{i}$. By Theorem 4.4, there is a continuous function $f$ on $\Delta_{i}$ which is the uniform limit of $\theta$-incomplete polynomials $\left\{P_{n}\right\}_{n=1}^{\infty}$ and is nonzero at $x_{0}$. Restricting to $\Sigma$ completes our argument.

We can use Theorem 4.5 to immediately produce a class of sets $\Sigma$ for which $Z_{\theta}=\theta^{2} \Sigma$. Let $K$ be a symmetric convex compact set in $\mathbb{R}^{k}$. We assume $K$ is symmetric in each coordinate, that is, if $\left(x_{1}, \ldots, x_{k}\right) \in K$ then $\left( \pm x_{1}, \ldots, \pm x_{k}\right) \in K$. Due to convexity, at every boundary point of $K$ there exists at least one supporting hyperplane to $K$. Since the class of $\theta$-incomplete polynomials is rotation invariant it suffices to state our lemma for the nonnegative orthant of $\mathbb{R}^{k}$.

LEMMA 4.6. Let $\Sigma=K \cap\left(\mathbb{R}^{+}\right)^{k}$ where $K$ is a symmetric convex compact set in $\mathbb{R}^{k}$. Then $\Sigma$ is an arbitrary intersection of $k$-simplices.

Proof. Let $\gamma \in \Sigma$ be a boundary point of $K$ such that there exists a supporting hyperplane $H_{\gamma}$ to $K$ at $\gamma$ which is not parallel to any of the coordinate hyperplanes $\left\{x \in \mathbb{R}^{k}: x_{j}=0\right\}$ where $j=1, \ldots, k$. Observe 
that $H_{\gamma}$ will also be a supporting hyperplane to $\Sigma$ at $\gamma$. Then the compact set $\Delta_{\gamma}$ bounded by the coordinate hyperplanes $\left\{x \in \mathbb{R}^{k}: x_{j}=0\right\}$ for $j=1, \ldots, k$ and $H_{\gamma}$ will be a $k$-simplex. Moreover, by definition, it contains $\Sigma$. We continue this procedure of constructing $k$-simplices, using all supporting hyperplanes to $\Sigma$ which are not parallel to coordinate hyperplanes. Taking the intersection of the corresponding $k$-simplices gives the appropriate expression for $\Sigma$. Note that by construction each $\Delta_{\gamma}$ contains $\Sigma$, so their intersection will also contain $\Sigma$. Equality is a consequence of the use of supporting hyperplanes to $\Sigma$.

Since a $k$-simplex in $\mathbb{R}^{k}$ is a compact section, combining the previous two results immediately gives

Proposition 4.7. Let $\Sigma=K \cap\left(\mathbb{R}^{+}\right)^{k}$ where $K$ is defined in the previous lemma. Then for a fixed $\theta$, we have $Z_{\theta}=\theta^{2} \Sigma$. In other words, in order for a continuous function $f$ on $\Sigma$ to be the uniform limit of $\theta$-incomplete polynomials it is necessary and sufficient for $f$ to vanish on $\theta^{2} \Sigma$.

We conclude this paper with a conjecture. If $K$ is a convex compact symmetric set in $\mathbb{R}^{k}$ with nonempty interior then $Z_{\theta}=\theta K$. That is, it will be possible to uniformly approximate a continuous function $f$ on $K$ by $\theta$-incomplete polynomials if and only if $f \equiv 0$ on $\theta K$.

\section{References}

[1] T. Bloom and N. Levenberg, Weighted pluripotential theory in $\mathbb{C}^{N}$, Amer. J. Math. 125 (2003), 57-103.

[2] M. von Golitschek, Approximation by incomplete polynomials, J. Approx. Theory 28 (1980), 155-160.

[3] M. Klimek, On the invariance of the L-regularity under holomorphic mappings, Zeszyty Nauk. Uniw. Jagielloń. Prace Mat. 23 (1982), 27-38.

[4] -, Pluripotential Theory, London Math. Soc. Monogr. 6, Oxford Univ. Press, 1991.

[5] A. Kroó, On approximation by bivariate incomplete polynomials, Constr. Approx. 10 (1994), 197-206.

[6] A. B. J. Kuijlaars, A note on weighted polynomial approximation with varying weights, J. Approx. Theory 87 (1996), 112-115.

[7] G. G. Lorentz, Problems for incomplete polynomials, in: Approximation Theory, III (Austin, TX, 1980), Academic Press, New York, 1980, 41-73.

[8] H. N. Mhaskar and E. B. Saff, Where does the $L^{p}$-norm of a weighted polynomial live?, Trans. Amer. Math. Soc. 303 (1987), 109-124.

[9] E. B. Saff and V. Totik, Logarithmic Potentials with External Fields, Grundlehren Math. Wiss. 316, Springer, Berlin, 1997. (Appendix B by T. Bloom).

[10] E. B. Saff and R. S. Varga, The sharpness of Lorentz's theorem on incomplete polynomials, Trans. Amer. Math. Soc. 249 (1979), 163-186.

[11] J. Siciak, Extremal plurisubharmonic functions in $\mathbb{C}^{n}$, Ann. Polon. Math. 39 (1981), $175-211$. 
[12] V. Totik, Weighted Approximation with Varying Weight, Lecture Notes in Math. 1569, Springer, Berlin, 1994.

Department of Mathematics

University of Toronto

Toronto, Canada M5S 3G3

E-mail: branker@math.toronto.edu

Reçu par la Rédaction le 22.3.2005

Révisé le 23.4.2005 